\title{
Fine jet structure of electrically charged grains in Enceladus' plume
}

\author{
G. H. Jones, ${ }^{1,2}$ C. S. Arridge, ${ }^{1,2}$ A. J. Coates, ${ }^{1,2}$ G. R. Lewis, ${ }^{1,2}$ S. Kanani, ${ }^{1,2}$
}

A. Wellbrock, ${ }^{1,2}$ D. T. Young, ${ }^{3}$ F. J. Crary ${ }^{3}$ R. L. Tokar, ${ }^{4}$ R. J. Wilson, ${ }^{4}$ T. W. Hill, ${ }^{5}$

R. E. Johnson, ${ }^{6}$ D. G. Mitchell, ${ }^{7}$ J. Schmidt, ${ }^{8}$ S. Kempf, ${ }^{9}$ U. Beckmann, ${ }^{9}$ C. T. Russell, ${ }^{10}$

Y. D. Jia, ${ }^{10}$ M. K. Dougherty, ${ }^{11}$ J. H. Waite Jr., ${ }^{3}$ and B. A. Magee ${ }^{3}$

Received 24 March 2009; revised 22 June 2009; accepted 27 July 2009; published 29 August 2009.

[1] By traversing the plume erupting from high southern latitudes on Saturn's moon Enceladus, Cassini orbiter instruments can directly sample the material therein. Cassini Plasma Spectrometer, CAPS, data show that a major plume component comprises previously-undetected particles of nanometer scales and larger that bridge the mass gap between previously observed gaseous species and solid icy grains. This population is electrically charged both negative and positive, indicating that subsurface triboelectric charging, i.e., contact electrification of condensed plume material may occur through mutual collisions within vents. The electric field of Saturn's magnetosphere controls the jets' morphologies, separating particles according to mass and charge. Fine-scale structuring of these particles' spatial distribution correlates with discrete plume jets' sources, and reveals locations of other possible active regions. The observed plume population likely forms a major component of high velocity nanometer particle streams detected outside Saturn's magnetosphere. Citation: Jones, G. H., et al. (2009), Fine jet structure of electrically charged grains in Enceladus' plume, Geophys. Res. Lett., 36, L16204, doi:10.1029/2009GL038284.

\section{Introduction}

[2] The Enceladus plume's primary components are water vapor [Hansen et al., 2006; Waite et al., 2006] and icy grains [Spahn et al., 2006; Porco et al., 2006]. Although the gas emerges at $\geq 600 \mathrm{~m} \mathrm{~s}^{-1}$ [Hansen et al., 2008], most grains are believed to emerge slower than Enceladus's $207 \mathrm{~m} \mathrm{~s}^{-1}$ escape velocity, thus falling back to the surface

\footnotetext{
${ }^{1}$ Mullard Space Science Laboratory, Department of Space and Climate Physics, University College London, Dorking, UK.

${ }^{2}$ Centre for Planetary Sciences at UCL/Birkbeck, London, UK.

${ }^{3}$ Space Science and Engineering Division, Southwest Research Institute, San Antonio, Texas, USA.

${ }^{4}$ Los Alamos National Laboratory, Los Alamos, New Mexico, USA.

${ }^{5}$ Physics and Astronomy Department, Rice University, Houston, Texas, USA.

${ }^{6}$ Department of Materials Science and Engineering, University of Virginia, Charlottesville, Virginia, USA.

Johns Hopkins University Applied Physics Laboratory, Laurel, Maryland, USA.

${ }^{8}$ AG Nichtlineare Dynamik, Universität Potsdam, Potsdam, Germany.

${ }^{9}$ Max Planck Institut für Kernphysik, Heidelberg, Germany.

${ }^{10}$ Institute of Geophysics and Planetary Physics, University of California, Los Angeles, California, USA.

${ }^{11}$ Blackett Laboratory, Imperial College London, London, UK.
}

Copyright 2009 by the American Geophysical Union. 0094-8276/09/2009GL038284
[Porco et al., 2006; Schmidt et al., 2008]. This velocity disparity was suggested to result from the condensation of water within surface fissures: wall collisions decelerate grains before emergence [Schmidt et al., 2008]. This condensation model predicts the formation of grains on scales from $\mathrm{nm}$ to tens of $\mu \mathrm{m}$, with the smallest attaining velocities close to the gas speed. CAPS [Young et al., 2004] measures Saturn's plasma environment. It includes an electron spectrometer, ELS, measuring energy-to-charge (E/q) ratios of negative particles from $0.6 \mathrm{eV} / \mathrm{e}$ to $28.8 \mathrm{keV} / \mathrm{e}$, and an ion mass spectrometer, IMS, covering positive particles of $\mathrm{E} / \mathrm{q}$ from $1 \mathrm{eV} / \mathrm{e}$ to $50.3 \mathrm{keV} / \mathrm{e}$. Although designed for electrons, ELS also detects negative ions [Coates et al., 2007]. During close Enceladus encounters E3 (March 12, 2008) and E5 (October 9, 2008), the ELS and IMS apertures were oriented to encompass the ram direction, allowing direct sampling of plume material moving slowly relative to Enceladus.

\section{Observations}

[3] During these plume traverses, IMS and ELS detected both positively and negatively-charged ions at E/q values expected for ionized plume gases, less than a few $100 \mathrm{eV}$ [Tokar et al., 2009; Coates et al., 2009]. The encounters' most striking and unexpected features are however the extremely high $>\sim 1 \mathrm{keV} / \mathrm{q}$ count rates within the plume (Figure 1). These signatures were only detected by CAPS anodes nearest ram, indicating that their cause was indeed plume material, and not magnetospheric plasma.

[4] When ions enter either detector at known velocities, their measured kinetic energy per charge can be converted to mass-to-charge (M/q). Plume ions had velocities of 14.41 (E3) and $17.73 \mathrm{~km} \mathrm{~s}^{-1}$ (E5) in Cassini's frame. For particles possessing a single electron charge, E/q would be proportional to grain mass. The maximum detectable $\mathrm{M} / \mathrm{q}$ value is controlled by the maximum energy detectable by both instruments; the higher E5 velocity therefore resulted in a reduced $\mathrm{M} / \mathrm{q}$ range. The $>\sim 500 \mathrm{eV} / \mathrm{e}$ signatures imply that ELS detected negative ions of M/q from 400 up to 26600 and $17600 \mathrm{amu} / \mathrm{q}$, respectively, and IMS detected positive ions up to 46500 and $30700 \mathrm{amu} / \mathrm{q}$. The grains' charge state is undetermined, but if they are singly-charged, they measure up to $>2 \mathrm{~nm}$ radius (Figure 2), i.e., orders of magnitude smaller than plume grains detected by other instrumentation [Spahn et al., 2006; Srama et al., 2004; Tokar et al., 2009].

[5] Figure 3 shows both encounters' nanograin signatures. In E3 IMS data, one clear, relatively broad flux peak is observed (E3il in Figure 3), and a minor isolated peak (E3i2). In ELS, two main peaks are seen: E3e1, detected over ELS's entire energy range and all anodes, possibly 

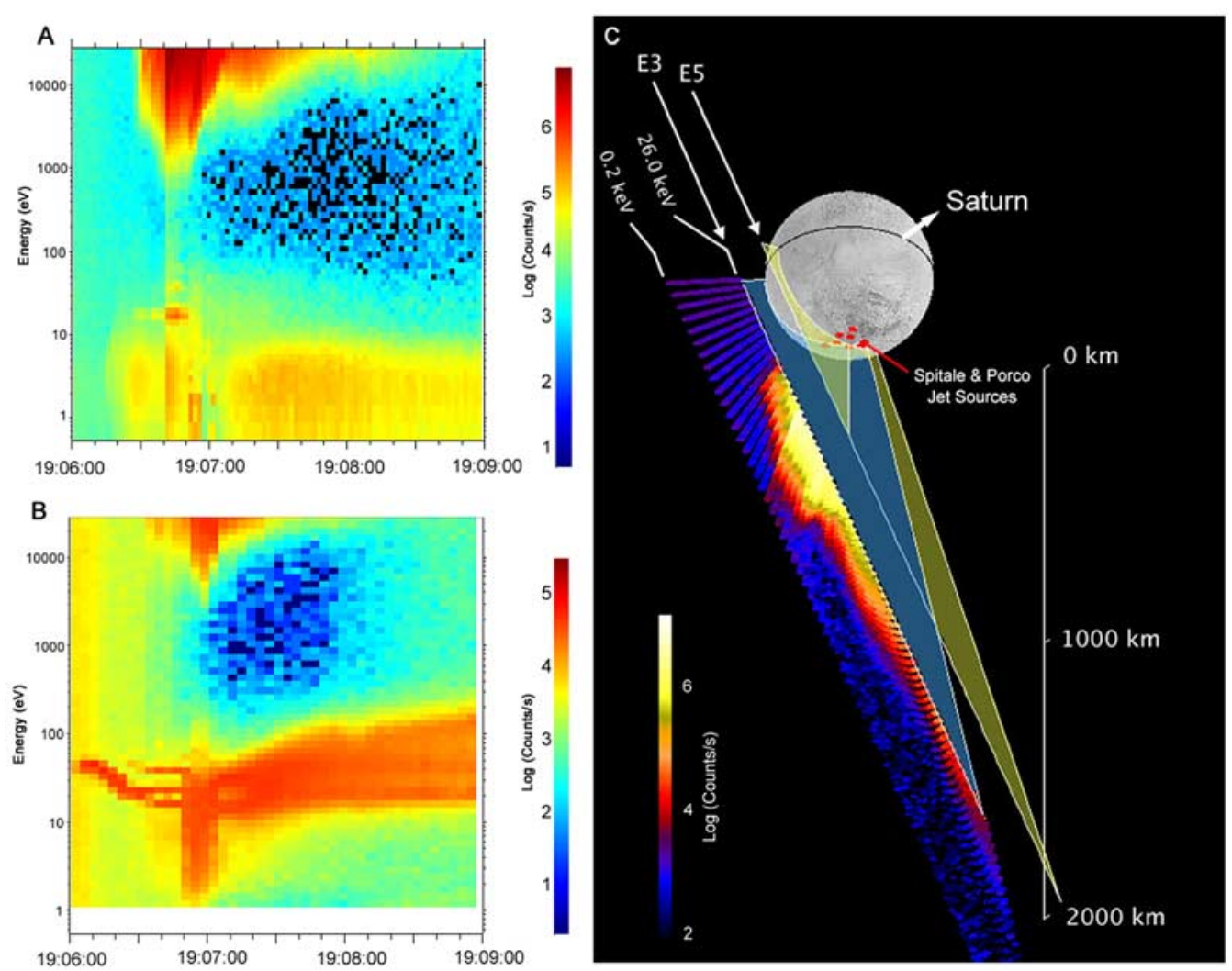

Figure 1. (a) ELS and (b) IMS spectrograms from the March 12, 2008 (E3) encounter of 504 km-wide Enceladus. The instruments have full energy scan resolutions of 2 and $4 \mathrm{~s}$, and energy sample cadences of 31.25 and $62.50 \mathrm{~ms}$. Complicating the spectrograms are changes in background penetrating radiation levels, caused by the shielding of Cassini from energetic magnetospheric particles by Enceladus itself and plume material [Jones et al., 2006]. Low energy negative ions in spectrogram in Figure 1a are described elsewhere [Tokar et al., 2009]. (c) The E3 (blue) and E5 (olive) trajectories past Enceladus from 0 to $2000 \mathrm{~km}$ south of the equatorial plane. The ground tracks intersect at the south pole. Closest approach occurred at 19:06:12 and 19:06:40 UT, respectively, at altitudes of 52 and $25 \mathrm{~km} .200 \mathrm{eV}-26.0 \mathrm{keV}$ data from ELS are shown along the E3 trajectory, with E/q decreasing with increasing distance from Enceladus. Enceladus's Hill sphere, where its gravitational influence is dominant, extends $\sim 948 \mathrm{~km}$ from the moon's center. CAPS observations of nanometer particles extended well beyond this boundary. All material detected outside must have exceeded escape velocity; some of this could be a grain population remaining near Enceladus once in free orbit about Saturn itself (S. Kempf, U. Beckmann, and J. Schmidt, personal communication, 2009).

resulting from grain fragmentation inside ELS, and E3e2, a complex signature where the peak energy shifts over time, indicating negative grains' spatial separation.

[6] CAPS detected both positively and negatively charged nanometer particles. The overriding charging process near Enceladus is caused by immersion in Saturn's corotational magnetospheric plasma, resulting in negative potentials [Kempf et al., 2006]. The co-existence of both populations at first appears anomalous. At nanometer scales, however, the charging process can be stochastic, depending on local plasma parameters. In addition, during their subsurface formation in a collisional environment [Schmidt et al., 2008], grains could undergo triboelectric charging: those that condense within the vent even when of the same composition, can acquire opposite charges. Smaller particles tend to charge negative, and larger ones positive [e.g., Duff and Lacks, 2008]. Most entrained nanograins are likely to have been accelerated to near-gas velocities. Overall, during both encounters CAPS detected negative particles to much lower kinetic energies than for positive particles; if a proxy for lower masses, this supports the picture of triboelectric charging occurring within vents. Although sunlight will have little effect in the nearEnceladus environment, particles' charge state changes could vary once exposed to the plume and the corotational plasma flow, where plasma parameters can differ significantly.

[7] During both traversals, nanograin particle fluxes were highly variable, especially at low altitudes, when the groundtrack speed was highest. The flux peaks are interpreted as Cassini's passage through or near collimated jets' cores. E5 IMS data were more highly-structured than E3, showing two strong, localized peaks (E5i1, E5i2), and a less obvious peak (E5i3). ELS peaks were numerous but more restricted in altitude than E3, possibly due to the reduced maximum detectable $\mathrm{M} / \mathrm{q}$ during E5, but may also indicate lower activity during E5. A double-energy ELS peak was observed at 19:06:55 (E5e2; Figures 2b and 3c). Although a discrete $\mathrm{E} / \mathrm{q}$ peak is possible, given the high along-track 

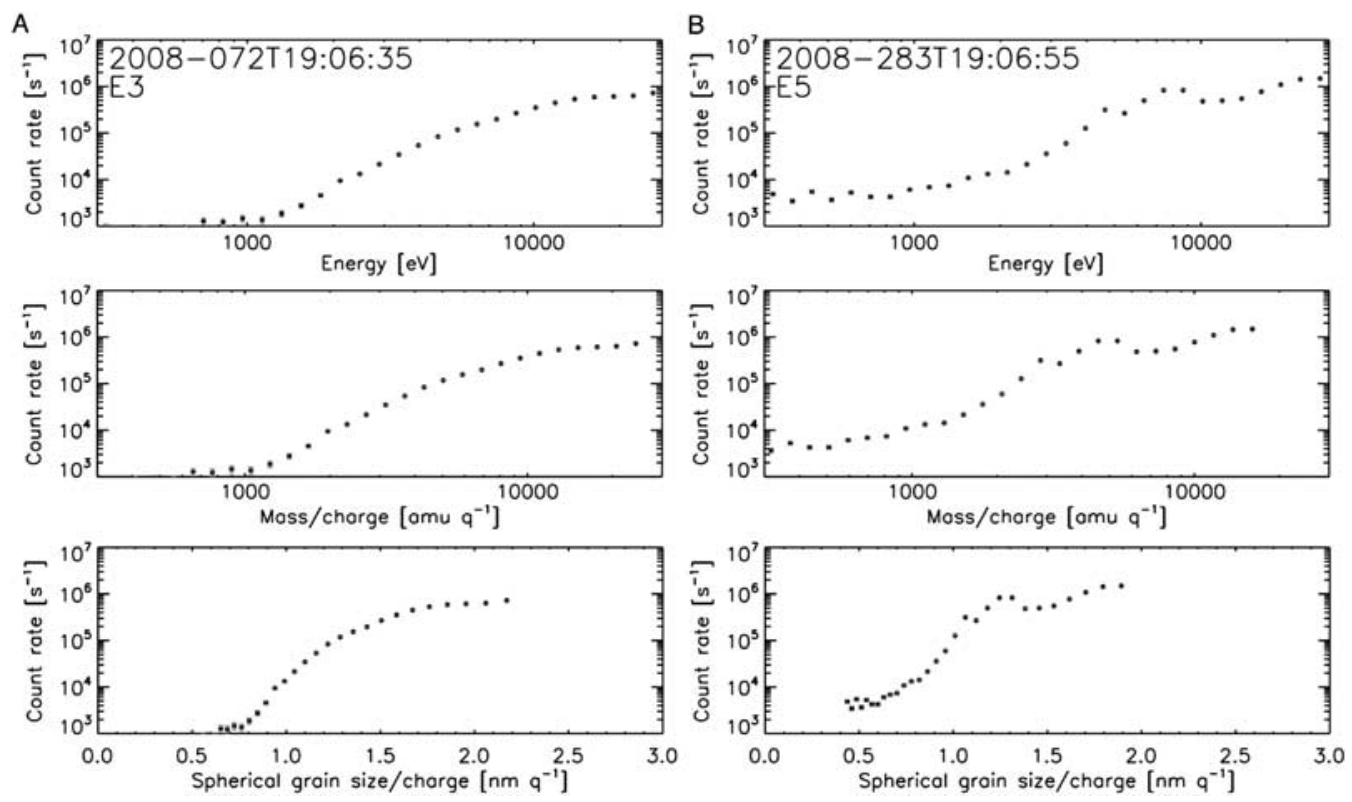

Figure 2. Two sample particle distributions obtained during (a) E3 and (b) E5. In both cases, (top) the E/q distribution is converted to $\mathrm{M} / \mathrm{q}$ assuming the given ram velocity, and finally to a radius per charge distribution, assuming spherical grains, and water ice density of $930 \mathrm{~kg} \mathrm{~m}^{-3}$. This density is appropriate for ice with a hexagonal crystal structure at $110-150 \mathrm{~K}$ [Lide, 2006]. However the ice crystal structure may differ: possible ice densities represent errors on these grain sizes small compared to the plotted points. The actual charge states are unknown; the inferred sizes therefore represent the minimum actual size if the grains possess a single electron charge. If grain structures are fractal, they can attain a relatively lower $\mathrm{M} / \mathrm{q}$ ratio than smaller particles; the slopes in E/q and hence M/q could therefore be affected by the grains' charge states and their masses.

speed, this is more likely explained by aliasing effects in ELS energy scans (scan rates restrict ELS to a $553 \mathrm{~m}$ alongtrack resolution during E5). This double peak occurred while crossing an unnamed extension to Camphor Sulcus (labelled on Figure 3a), then Alexandria Sulcus. We note its coincidence with a high relative fault activity region in models of shear failure [Smith-Konter and Pappalardo, 2008], and the consequent possibility of it being a previously-unidentified jet. Both encounters show relatively abrupt starts to the high-energy signatures, at latitudes of $\sim 61^{\circ}$ and $\sim 66^{\circ}$ south, at altitudes of $\sim 150$ and $\sim 100 \mathrm{~km}$, respectively. This is considerably farther north than identified jet sources [Spitale and Porco, 2007], and if not due to localized sources near these latitudes, may represent downfalling material.

\section{Charge Separation of Oppositely-Charged Grains}

[8] During both E3 and E5, there are notable differences in the timings of peaks observed by ELS and IMS (Figure 3), i.e., in negative and positive particle fluxes. A simple comparison of sources and ELS peaks suggests a strong correlation for E3, and a weaker one for E5 (Figure 4). The separation of peak particle fluxes by charge indicates that nanograins of similar $\mathrm{M} / \mathrm{q}$ have been deflected in different directions according to their charge states. The local motional electric field $(\underline{E})$ is generally directed anti-Saturnward and initially accelerates positively (negatively) charged grains away from (towards) the planet. Once accelerated radially, Lorentz and gravitational forces induce azimuthal deflection producing cycloidal trajectories, accelerating particles generally in the orthogonal, corotation direction. However, the plume's presence significantly affects local plasma motion. Corotational flow is slowed and deflected near Enceladus due to mass-loading by plume ions [Tokar et al., 2006, 2009]. In a near-stagnant flow, the accelerating force will be weak; the gyroradii of the detected particles could be as small as $0.2-1 \mathrm{~km}$, assuming near-complete flow stagnation. Flow deflection would rotate the motional electric field in the plane of the figure, and the magnetic field's slight deviation from a north-south orientation [Dougherty et al., 2006] will induce north-south electric field components.

[9] Accounting for particle motions under the generalized Lorentz force directions, we can associate most E3 and E5 flux peaks with jet and fracture locations (Table 1). We suggest that a local, as yet unseen source may exist in the region of E3e5. Although by separating the charged populations, the Lorentz force clearly affects the grains, the clearly linked nanograin flux peaks and observed jets suggest that the force is relatively weak, in turn indicating that the plasma flow approaches complete stagnation within the plume.

[10] Simulations of charged grains' trajectories in the CAPS $\mathrm{M} / \mathrm{q}$ range indicate that each jet will split into two components on either side of the flow axis, with negative particles Saturnward. Low M/q particles remain in tight streams, but are deflected downstream at the lowest alti- 

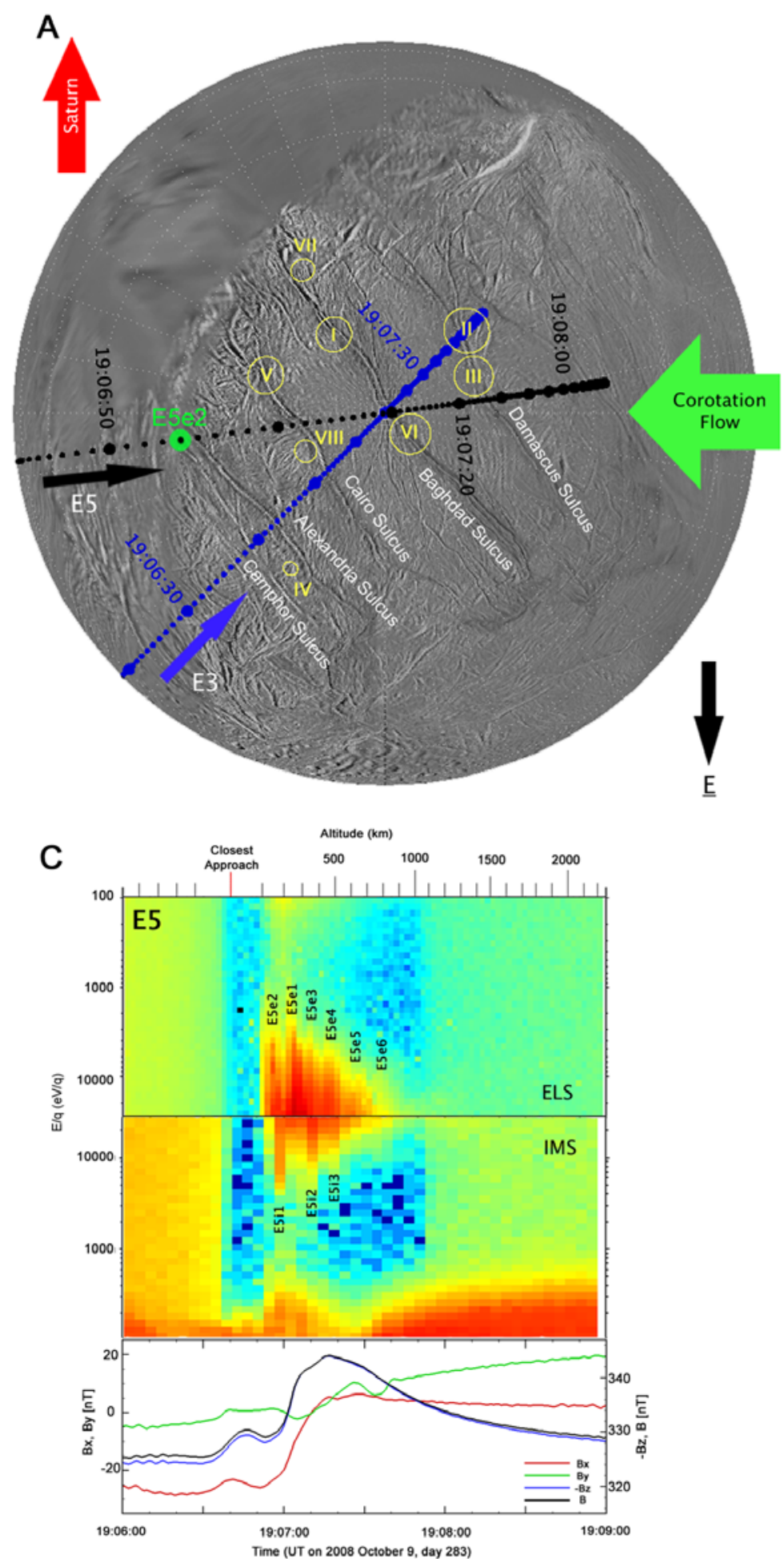

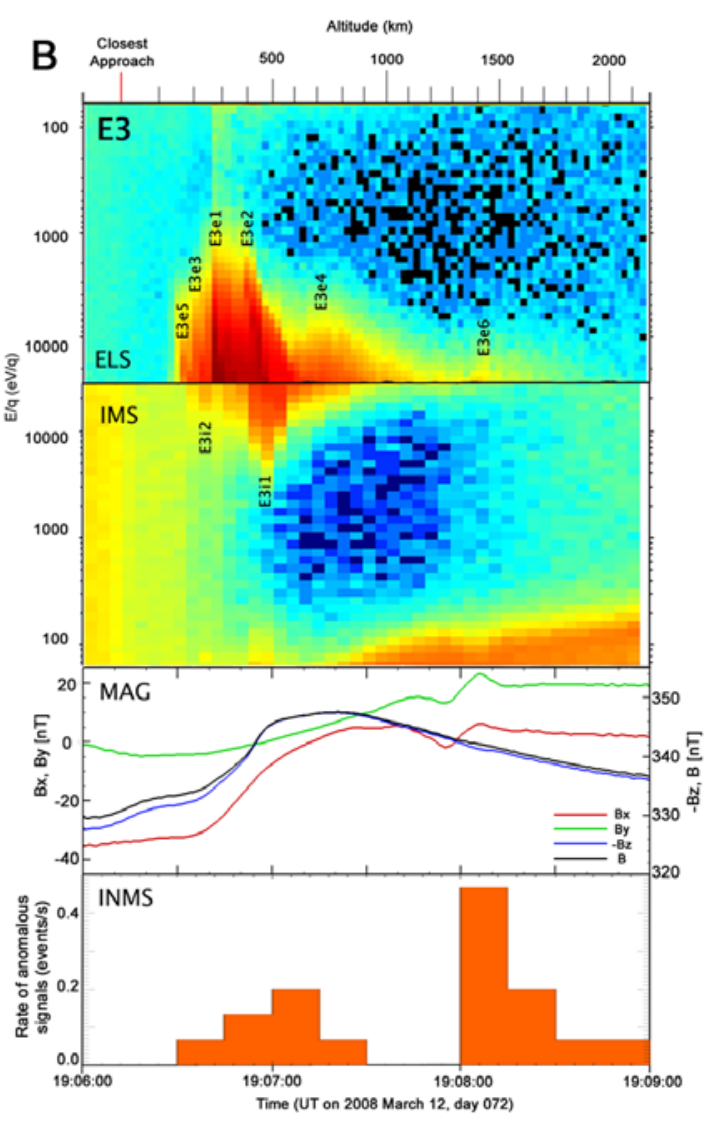

Figure 3. Ground track of Cassini, observed nanograin fluxes and magnetic field observations. (a) Cassini's E3 and E5 paths projected onto Enceladus's surface [Roatsch et al., 2008]; times are in UT, with large symbols every $10 \mathrm{~s}$. The view is centered on Enceladus's south pole; the direction towards Saturn, and the magnetospheric corotational flow are arrowed. Regions labelled with roman numerals denote jet sources identified from imaging observations [Spitale and Porco, 2007]; each circle corresponds to the 1-sigma error on their locations. Electromagnetic forces affect charged grains' trajectories. (b and c) E/q spectrograms obtained by (top) ELS and (bottom) IMS during E3 and E5. The ELS E/q range has been reversed to better show relative timings of features. Peaks have been labelled according to encounter (E3 or E5), instrument (e/i) and relative peak strength $(1,2 \ldots)$, e.g., E5i2 is the second-strongest IMS peak during E5. Third plots show the Cartesian magnetic field components and magnitude [Dougherty et al., 2004]; the right-handed system has X oriented in the corotational flow direction, and Y towards Saturn's center. Anomalous INMS data are also shown for E3. 

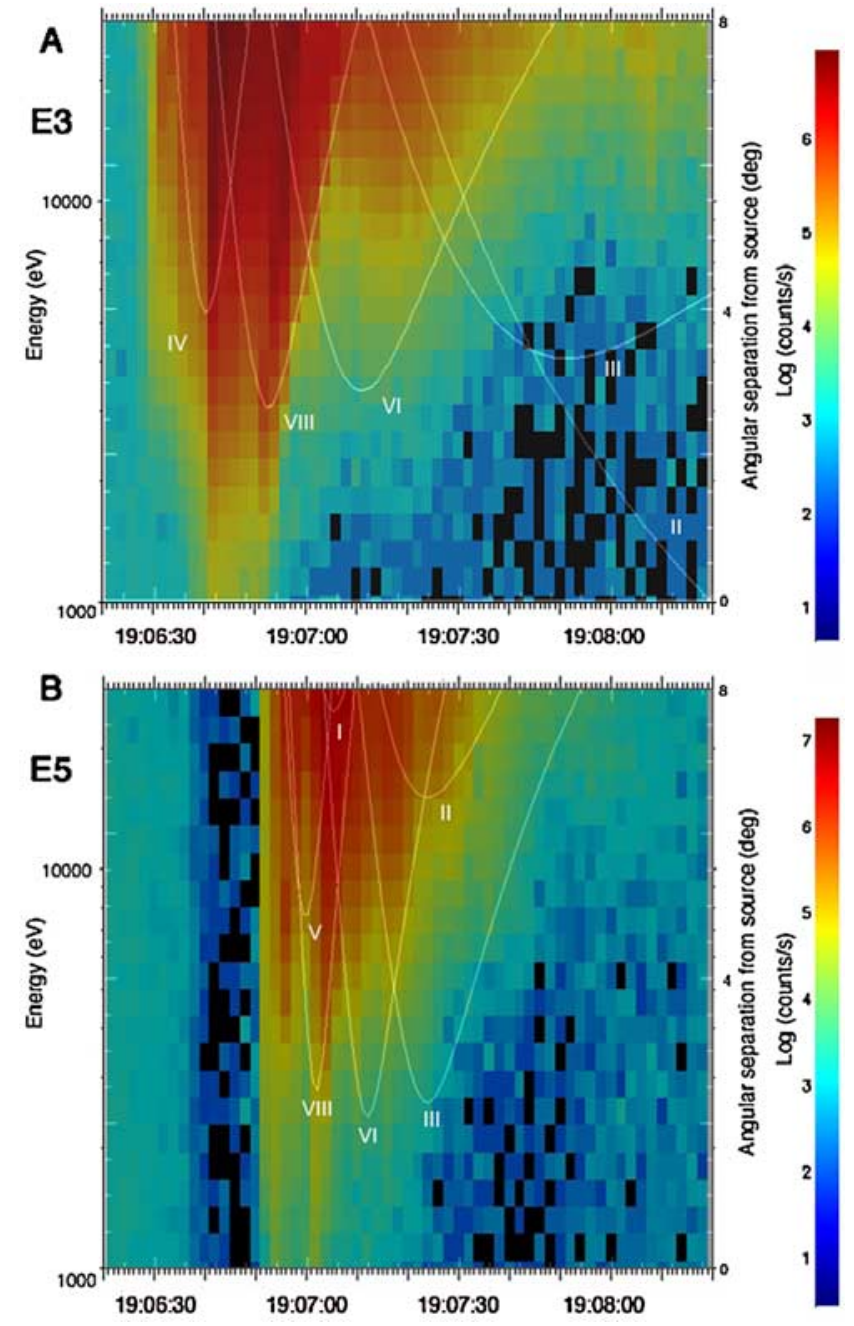

Figure 4. Correspondence between ELS spectrograms and jet sources. (a) E3 and (b) E5 spectrograms; overlaid in white is the angular separation of Cassini from the centre of each known jet source (I-VIII) [Spitale and Porco, 2007]; scale at right. There is excellent correspondence between expected peaks and those observed during E3, except for source III, which is known to be oriented away from Cassini's trajectory. The use of angular separations from sources means that the non-vertical but impreciselyconstrained flow directions of some jets have been ignored, and vertical jet orientations assumed. For E3, the agreement was good, but was moderate for E5. tudes. Although the higher mass population is more spatially extended due to larger gyroradii, its constituents remain closest to uncharged grains' expected trajectories to the highest altitudes. The positively-charged population that can be accelerated outwards by the magnetospheric corotational electric field is very likely a major component of the dust streams observed outside Saturn's magnetosphere [Kempf et al., 2005].

\section{Discussion and Conclusion}

[11] We note the correspondence between some CAPS flux peaks and magnetic field deviations measured by Cassini's magnetometer [Dougherty et al., 2004]. Particularly notable around E3e6 ( 19:08:00) is a strong localized field deflection. This also coincides with a brief decrease in IMS ion velocities, observable at the bottom of the IMS spectrogram (Figure 3b). Furthermore, in Ion and Neutral Mass Spectrometer [Waite et al., 2004] data, these features coincide with a local peak in the frequency of anomalous spikes in the time signatures of mass peaks representing water, carbon dioxide, and simple organics. These rapid density changes are interpreted as resulting from micronsized grains entering and vaporizing inside the instrument antechamber. During the E3 encounter, approximately $20 \%$ of the registered anomalous spikes occurred for seven different masses within a $30 \mathrm{~s}$ time span, correlating with the secondary peak seen at 19:08:09.

[12] The correlation of perturbations at least suggests a spatial coincidence in nanograin particle concentrations and localized changes to the magnetospheric plasma, and we associate this particular feature (E3e6), with source II on Damascus Sulcus. The possibility exists that the charged grains themselves play a part in inducing the plasma and magnetic field signatures, possibly by forming a tightlyconstrained mass-loading source. A magnetic field deviation of similar scale occurs from 19:07:25 onwards during E5, coinciding with a weak ELS flux enhancement, possibly linked to source III.

[13] We have detected a population within the Enceladus plume material that spans the mass range between gaseous material and previously-detected, larger grains. The population's electrical charge and other characteristics may hold important clues to subsurface conditions. The observable effects of collimated jets indicate that the plume's fine structure exerts a significant effect on Saturn's magnetosphere well beyond Enceladus's gravitational sphere of influence.

Table 1. Detected Flux Peaks and Their Inferred Likely Source(s), Where Identified

\begin{tabular}{cccc}
\hline E3 Flux Peak & Inferred Source(s) & E5 Flux Peak & Inferred Source(s) \\
\hline E3e5 & Unknown & E5e2 & Unnamed Sulcus and Alexandria Sulcus \\
E3e3 & E5e1 & Cairo Sulcus: VIII \\
E3e1 & Ennamed Sulcus (north of Camphor Sulcus) & E5e3 & Baghdad Sulcus: VI \\
E3e2 & Alexandria Sulcus: IV & E5e4 & Baghdad Sulcus: second source? \\
E3e4 & Cairo Sulcus: linked to VIII? & Damascus Sulcus \\
E3e6 & Baghdad Sulcus: VI & E5e6 (weak) & Damascus Sulcus: III \\
E3i2 & Damascus Sulcus: II, possibly III & E5i1 & Cairo Sulcus: V \\
E3i1 & Camphor Sulcus & E5i2 & Baghdad Sulcus: linked to VI? \\
& Cairo Sulcus: VIII & E5i3 & Damascus Sulcus \\
\hline
\end{tabular}


[14] Acknowledgments. Cassini-Huygens is a mission of international collaboration between NASA, the European Space Agency (ESA), and the Agenzia Spaziale Italiana (ASI). Cassini research at MSSL-UCL is supported by the UK Science and Technology Research Council, STFC. GHJ is supported by an STFC Advanced Fellowship. Cassini research in the United States is supported by NASA/JPL contract 1243218 with Southwest Research Institute.

\section{References}

Coates, A. J., et al. (2007), Discovery of heavy negative ions in Titan's ionosphere, Geophys. Res. Lett., 34, L22103, doi:10.1029/ 2007GL030978.

Coates, A. J., G. H. Jones, G. R. Lewis, A. Wellbrock, D. T. Young, F. J. Crary, R. E. Johnson, T. A. Cassidy, T. W. Hill (2009), Negative Ions in the Enceladus Plume, Icarus, in press.

Dougherty, M. K., et al. (2004), The Cassini magnetic field investigation, Space Sci. Rev., 114, 331-383, doi:10.1007/s11214-004-1432-2.

Dougherty, M. K., et al. (2006), Identification of a dynamic atmosphere at Enceladus with the Cassini magnetometer, Science, 311, 1406-1409, doi: $10.1126 /$ science. 1120985

Duff, N., and D. J. Lacks (2008), Particle dynamics simulations of triboelectric charging in granular insulator systems, J. Electrost., 66, 51-57, doi:10.1016/j.elstat.2007.08.005.

Hansen, C. J., et al. (2006), Enceladus' water vapor plume, Science, 311, $1422-1425$, doi:10.1126/science.1121254.

Hansen, C. J., et al. (2008), Water vapour jets inside the plume of gas leaving Enceladus, Nature, 456, 477-479, doi:10.1038/nature07542.

Jones, G. H., et al. (2006), Enceladus' varying imprint on the magnetosphere of Saturn, Science, 311, 1412-1415, doi:10.1126/science. 1121011.

Kempf, S., et al. (2005), High-velocity streams of dust originating from Saturn, Nature, 433, 289-291, doi:10.1038/nature03218.

Kempf, S., et al. (2006), The electrostatic potential of E ring particles, Planet. Space Sci., 54, 999-1006, doi:10.1016/j.pss.2006.05.012.

Lide, D. R. (Ed.) (2006), CRC Handbook of Chemistry and Physics, 87th ed., Taylor and Francis, London.

Porco, C. C., et al. (2006), Cassini observes the active south pole of Enceladus, Science, 311, 1393-1401, doi:10.1126/science.1123013.

Roatsch, T., et al. (2008), High-resolution Enceladus atlas derived from Cassini-ISS images, Planet. Space Sci., 56, 109-116, doi:10.1016/ j.pss.2007.03.014.

Schmidt, J., N. Brilliantov, F. Spahn, and S. Kempf (2008), Slow dust in Enceladus' plume from condensation and wall collisions in tiger stripe fractures, Nature, 451, 685-688, doi:10.1038/nature06491.

Smith-Konter, B., and R. T. Pappalardo (2008), Tidally driven stress accumulation and shear failure of Enceladus' tiger stripes, Icarus, 198, 435451, doi:10.1016/j.icarus.2008.07.005.

Spahn, F., et al. (2006), Cassini dust measurements at Enceladus and implications for the origins of the E ring, Science, 311, 1416-1418, doi:10.1126/science.1121375.
Spitale, J. N., and C. C. Porco (2007), Association of the jets of Enceladus with the warmest regions on its south-polar fractures, Nature, 449, 695-697, doi:10.1038/nature06217.

Srama, R., et al. (2004), The Cassini Cosmic Dust Analyzer, Space Sci. Rev., 114, 465-518, doi:10.1007/s11214-004-1435-z.

Tokar, R. L., et al. (2006), The interaction of the atmosphere of Enceladus with Saturn's plasma, Science, 311, 1409-1412, doi:10.1126/ science. 1121061.

Tokar, R. L., R. E. Johnson, M. F. Thomsen, R. J. Wilson, D. T. Young, F. J. Crary, A. J. Coates, G. H. Jones, and C. S. Paty (2009), Cassini detection of Enceladus' cold water-group plume ionosphere, Geophys. Res. Lett., 36, L13203, doi:10.1029/2009GL038923.

Waite, J. H., et al. (2004), The Cassini Ion and Neutral Mass Spectrometer (INMS) investigation, Space Sci. Rev., 114, 113-231, doi:10.1007/ s11214-004-1408-2.

Waite, J. H., et al. (2006), Cassini Ion and Neutral Mass Spectrometer: Enceladus plume composition and structure, Science, 311, 1419-1422, doi:10.1126/science.1121290.

Young, D., et al. (2004), Cassini Plasma Spectrometer investigation, Space Sci. Rev., 114, 1-112, doi:10.1007/s11214-004-1406-4.

C. S. Arridge, A. J. Coates, G. H. Jones, S. Kanani, G. R. Lewis, and A. Wellbrock, Mullard Space Science Laboratory, Department of Space and Climate Physics, University College London, Holmbury St. Mary, Dorking RH5 6NT, UK. (ghj@mssl.ucl.ac.uk)

U. Beckmann and S. Kempf, Max Planck Institut für Kernphysik, Saupfercheckweg 1, D-69117 Heidelberg, Germany.

F. J. Crary, B. A. Magee, J. H. Waite Jr., and D. T. Young, Space Science and Engineering Division, Southwest Research Institute, 6220 Culebra Road, San Antonio, TX 78238, USA.

M. K. Dougherty, Blackett Laboratory, Imperial College London, London SW7 2BW, UK.

T. W. Hill, Physics and Astronomy Department, Rice University, Box 1892, MS 108, 6100 South Main Street, Houston, TX 77005-1892, USA.

Y. D. Jia and C. T. Russell, Institute of Geophysics and Planetary Physics, University of California, Los Angeles, 6863 Slichter Hall, Los Angeles, CA 90095, USA.

R. E. Johnson, Department of Materials Science and Engineering, University of Virginia, P.O. Box 400745, 116 Engineer's Way, Charlottesville, VA 22904, USA.

D. G. Mitchell, Johns Hopkins University Applied Physics Laboratory, 11100 Johns Hopkins Road, Laurel, MD 20723, USA.

J. Schmidt, AG Nichtlineare Dynamik, Universität Potsdam, Postfach 601553, D-14469 Potsdam, Germany.

R. L. Tokar and R. J. Wilson, Los Alamos National Laboratory, Mail Stop D466, Los Alamos, NM 87545, USA. 